\title{
Novel Chemical Mechanical Polishing/Plasma- Chemical Vaporization Machining (CMP/P-CVM) Combined Processing of Hard-to-Process Crystals Based on Innovative Concepts
}

\author{
Toshiro K. Doi ${ }^{1, *}$, Yasuhisa Sano ${ }^{2}$, Syuhei Kurowaka ${ }^{3}$, Hideo Aida ${ }^{1,4}$, \\ Osamu Ohnishi ${ }^{5}$, Michio Uneda ${ }^{6}$ and Koki Ohyama ${ }^{4}$ \\ ${ }^{1}$ Kyushu University, KASTEC, 6-1 Kasuga-Koen, Kasuga-shi, Fukuoka 816-8580, Japan \\ ${ }^{2}$ Osaka University, 1-1 Yamadaoka, Suita-shi, Osaka 565-0871, Japan \\ ${ }^{3}$ Kyushu University, Ito Campus, 744 Motoka, Nishi-ku, Fukuoka 819-0395, Japan \\ ${ }^{4}$ Namiki Precision Jewel Co., Ltd., 3-8-22 Shinden, Adachi-ku, Tokyo 123-8511, Japan \\ ${ }^{5}$ Miyazaki University, 1-1 Gakuen Kibanadai-nishi, Miyazaki 889-2192, Japan \\ ${ }^{6}$ Kanazawa Institute of Technology, 7-1 Ohgigaoka, Nonoichi-shi, Ishikawa 921-8501, Japan
}

(Received February 10, 2014; accepted March 10, 2014)

Key words: chemical mechanical polishing (CMP), plasma-chemical vaporization machining (P-CVM), combined processing, hard-to-process crystals, pseudoradical area, removal rate, surface roughness, ultraprecision processing

In this research, we aim to establish systematic knowledge of ultraprecision processing of hard-to-process crystal wafers for next-generation "green devices", and design and develop a high-efficiency, high-quality process to contribute to an early commercialization of $\mathrm{SiC}, \mathrm{GaN}$, and diamond-based devices demanded for a low-carbon society. Upon designing an ultrahigh-precision process for hard-to-process materials, we divided the process into two steps: a pretreatment step and a finishing step. In the pretreatment step, a pseudoradical area was formed by introducing ultrafine defects to control the surface condition suitable for finishing. In the finishing step, we attempted to combine the closed-chamber chemical mechanical polishing (CMP) and plasma-chemical vaporization machining ( $\mathrm{P}-\mathrm{CVM})$ methods. To evaluate the concept of the second step, a fundamental study needed to design and prototype the machine was conducted. As a method of creating a pseudoradical area, femtosecond (fs) laser irradiation and coarse processing (grinding), which leaves a crystallographically disordered layer, were considered. Microindentation test, cross-sectional transmission electron microscopy (TEM) observation, Raman spectroscopy, X-ray photoelectron spectroscopy (XPS) and reflection high-energy electron diffraction (RHEED) were used to confirm the

${ }^{*}$ Corresponding author: e-mail: doi@astec.kyushu-u.ac.jp 
pseudoradical area at the uppermost surface. Also, we studied the characteristics of the pseudoradical substrates subjected to CMP and P-CVM. P-CVM showed an increased processing rate for the pseudoradical substrates having microdefects. It will be necessary to optimize the degree and depth of crystallographic disorder in the pseudoradical area in the future. On the basis of the results presented here, we are starting to prototype the innovative CMP/P-CVM combined processing machine, which can selectively flatten the nanotopographies of hard-to-process materials by conducting CMP and P-CVM while continuously creating a pseudoradical area through an in situ physical effect such as polishing.

\section{Introduction}

The recent trend of constructing highly functional systems with reduced energy consumption demands research and development of light-emitting diodes (LEDs) and power transistors using sapphire substrates for epitaxy, silicon carbide ( $\mathrm{SiC})$ and gallium nitride $(\mathrm{GaN}){ }^{(1)}$ "A $\mathrm{SiC} / \mathrm{GaN}$ device in the post-Si era" is coming and is followed by "an ultimate device using semiconductor diamond." $\mathrm{SiC}, \mathrm{GaN}$ and semiconductor diamonds are truly ideal materials since the poformance of high-power control devices, high-frequency, high-power devices, and switching devices fabricated using these semiconductor materials is ten to ten thousand times higher performance than that of $\mathrm{Si}$ based devices. In particular, single-crystalline semiconductor diamonds are expected to be used in future devices in 2020 or later, after the next-generation devices. ${ }^{(2)}$

Although semiconductor materials such as $\mathrm{SiC}$ and $\mathrm{GaN}$ for power devices and LEDs are highlighted, in this research, we aim to establish an innovative process for diamond substrates as part of a project to realize "an ultimate device by semiconductor diamond". The process for semiconductor diamond requires a damage-free mirrorlike surface of the hardest material on Earth with high throughput. The lack of an achieving ultrahighprecision process for semiconductor diamonds would become a technological bottleneck in realizing the ultimate device. However, it is difficult to apply the conventional processing technology to diamond owing to the extreme hardness and chemical stability of this material.

In this research, we aim to establish systematic knowledge of ultraprecision processing of hard-to-process crystal wafers for next-generation "green devices", and design and develop a high-efficiency, high-quality process to contribute to an early commercialization of $\mathrm{SiC}, \mathrm{GaN}$ and diamond-based devices demanded for a low-carbon society.

In this work, we propose a new concept of high-efficiency processing that utilizes a "pseudoradical area" created on a work surface of the substrate. A novel, high-efficiency, high-quality processing machine is designed by combining the closed-chamber chemical mechanical polishing (CMP)(3) and plasma-chemical vaporization machining (P-CVM) methods. ${ }^{(4)}$ We report results from various fundamental studies necessary to design and prototype the machine. 


\section{Proposed High-Efficiency Process: Basic Concept}

To design an ultraprecision process for hard-to-process materials, we divide the process into two steps: a pretreatment step and a finishing step. The pretreatment step primarily aims to create a pseudoradical area so that the surface condition becomes suitable for the finishing step. For the finishing step, we aim to design and prototype a machine combining a closed-chamber CMP method, which encloses a CMP setup with a chamber (bell jar) to control the process environment, and a P-CVM method, which uses atmospheric-pressure plasma of air or oxygen. Here, we consider two cases: (a) creating a pseudoradical area during the pretreatment step for easier finishing (we call the treated substrate in this case an ex situ pseudoradical substrate) and b) creating a pseudoradical area during the finishing step (we call this process an in situ pseudoradical processing).

The background and basic strategy of this research are presented in Figs. 1 and 2, respectively. In this research, we target the processing of a wafer surface after slicing and primarily aim to finish the wafer using the following two steps.

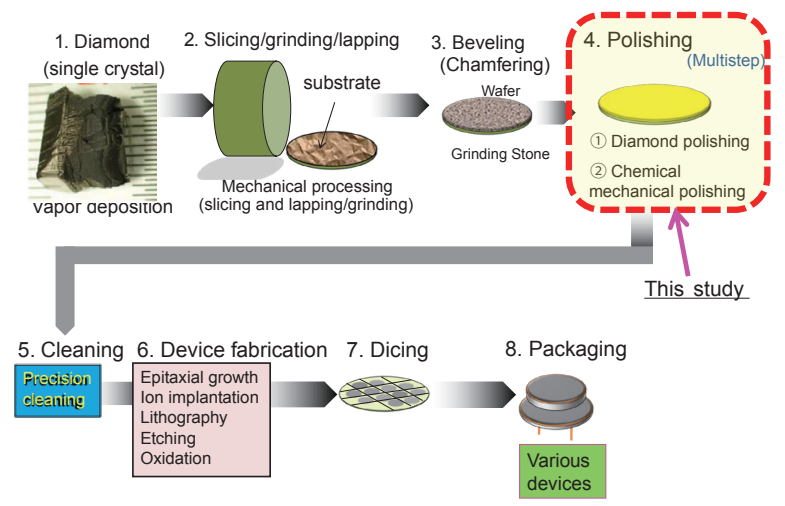

Fig. 1. (Color online) General process flow of hard-to-process materials.

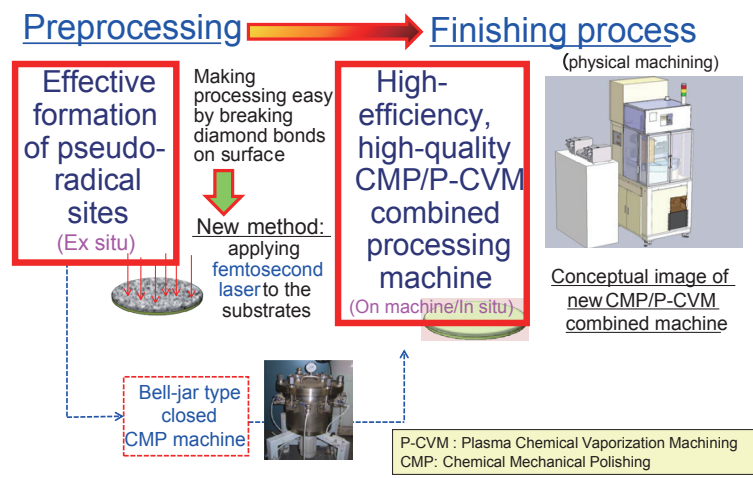

Fig. 2. (Color online) Proposed method of processing hard-to-process materials (e.g., diamond crystal). 
(1) Pretreatment step

By femtosecond (fs) laser irradiation on the surface of a hard-to-process substrate, we aim to create ultrafine defects (in an area we call the "pseudoradical area") at the very top of the surface (a few tens to a few hundreds of atomic layers). The purpose of this treatment is to make the surface suitable for CMP under atmospheric pressure by forming the laser-induced pseudoradical area on the entire surface, which would induce strong mechanochemical reactions between the pseudoradical area and the area of chemical reactions associated with the mechanical polishing and abrasion. Figure 3 shows a schematic drawing of the pseudoradical area obtained by fs laser irradiation. Utilizing the fs laser irradiation presented here is one example of creating a pseudoradical area, and we believe that other treatments that create ultrafine defects on the surface would be effective as well. In addition to the fs laser irradiation, in this paper, we outline a coarse process (mechanical grinding) that intentionally leaves a crystallographically disordered layer (process-induced damaged layer) on the surface.

The substrate pretreated in this manner corresponds to the above-defined ex situ pseudoradical substrate and will be processed in the finishing step explained below. We also assume that the current closed-chamber CMP (bell-jar-type CMP) method ${ }^{(5)}$ could also be used to finish the surface.

\section{(2) Finishing step}

We have developed a machinery technology based on the closed-chamber CMP and P-CVM methods. The former technology has attained a process efficiency that is a few times higher than that of a conventional CMP method by controlling the CMP environment with a chamber enclosure that can hold high-pressure gas. ${ }^{(6)}$ This method can achieve excellent flatness, although it requires further improvement of the process efficiency of hard-to-process materials.

In contrast, the P-CVM method, which employs atmospheric-plasma processing, can attain a higher process efficiency. It enables high-efficiency etching with high-density neutral radicals and does not induce plasma ion damage on the work surface owing to the low kinetic energy of ions. Moreover, this method has demonstrated a high etching rate on hard-to-process materials such as $\mathrm{SiC}$ and $\mathrm{GaN},{ }^{(7)}$ although it has difficulties in flattening the surface efficiently owing to isotropic etching by neutral radicals.

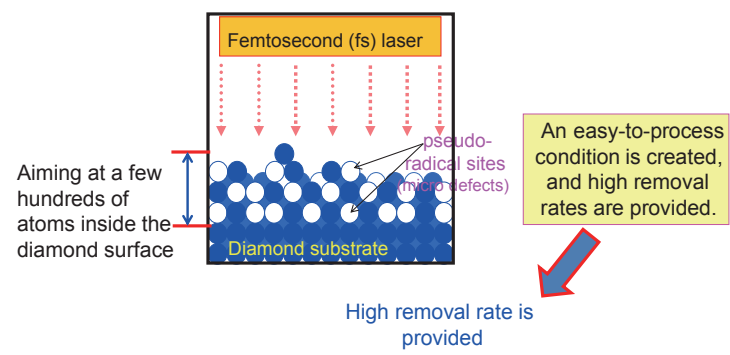

Fig. 3. (Color online) Formation model of pseudoradical sites using fs laser. 
In this research, we aim to propose and prototype a processing machine that enables high-efficiency, high-quality processing of hard-to-process materials by combining the closed-chamber CMP and P-CVM methods while taking advantage of the merits and mitigating the demerits of both methods.

This novel fusion machine flattens the surface by continuously creating a pseudoradical area during the process. A key part of the machine utilizes special pads and slurry to apply a physical effect and induce strong mechanochemical reactions between the pseudoradical area and the chemical reactions associated with the tribological ablation and polishing. This corresponds to the above-defined in situ pseudoradical processing. The machine can use high-pressure oxygen to enhance the etching effect of P-CVM. Figure 4 shows a schematic drawing of the novel fusion machine combining the closed-chamber CMP and P-CVM methods.

On the basis of the concept explained above, in this paper, we report a fundamental study on hard-to-process materials, which is needed to design and prototype the novel fusion machine.

\section{Experiment, Equipment, and Process Conditions}

A fundamental study was carried out with single-crystalline diamond as well as other single-crystalline substrates such as $\mathrm{SiC}$ and $\mathrm{GaN}$.

\subsection{Experiment for pretreatment step}

For the pretreatment step, a preliminary study on fs laser irradiation was conducted with single-crystal diamond synthesized under high pressure and high temperature.

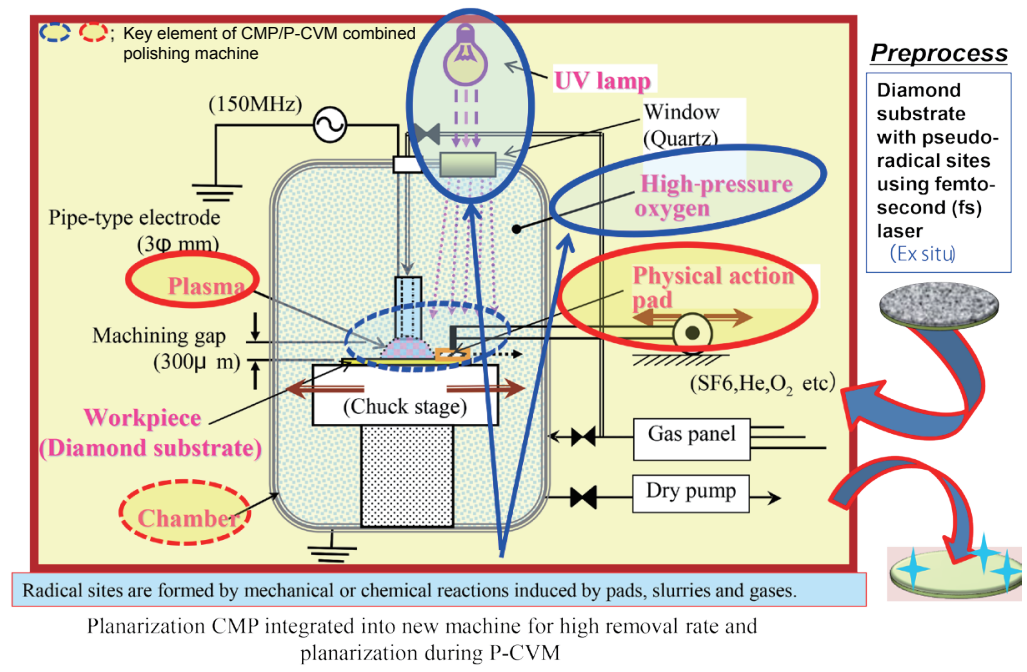

Fig. 4. (Color online) New concept of CMP/P-CVM combined processing machine. 
Sample diamond substrates for this experiment were prepared by flattening them by a dry polishing method using a special fixed diamond plate. As shown in Fig. 5, the processing rate greatly depends on the polishing orientation. The surface roughness is small at a low processing rate. By taking advantage of this nature, the starting samples for this experiment were prepared by flattening the substrates in the following two steps:

1) flattening by polishing along the $<100>$ direction,

2) polishing along the $<110>$ direction until no scratches are observed with the naked eyes.

The pseudoradical area was created on the prepared diamond substrates by irradiating the surface with a fs laser and its properties were evaluated. The laser wavelength was $1045 \mathrm{~nm}$ and the laser power was $10 \mathrm{~mW}$. The laser was scanned by moving the sample stage at $400 \mathrm{~mm} / \mathrm{s}$ and the irradiation pitch was $10 \mu \mathrm{m}$. Since the laser spot size was 30 $\mu \mathrm{m}$, the entire surface of the diamond substrate was irradiated with the fs laser.

The cross section of the irradiated sample was observed by transmission electron microscopy (TEM). A bonding state of carbon in the diamond was characterized by Raman spectroscopy for samples before and after laser irradiation. Additionally, a microindentation test was carried out for the diamond surface with and without a pseudoradical area.

We also carried out a finishing process on a sample with a pseudoradical area created by laser irradiation and on a sample subjected to mechanical polishing to confirm the effect of the pseudoradical area on the processing. The finishing process used was CMP at a rotation speed of $300 \mathrm{rpm}$ and a load of $1 \mathrm{~kg}$ using a felt pad on a plate $(\varphi 280 \mathrm{~mm})$ and a special oxidizing slurry. Surface roughness was measured from time to time during the finishing process and the effect of the pseudoradical area on the processing was evaluated from the time-dependent roughness curve.

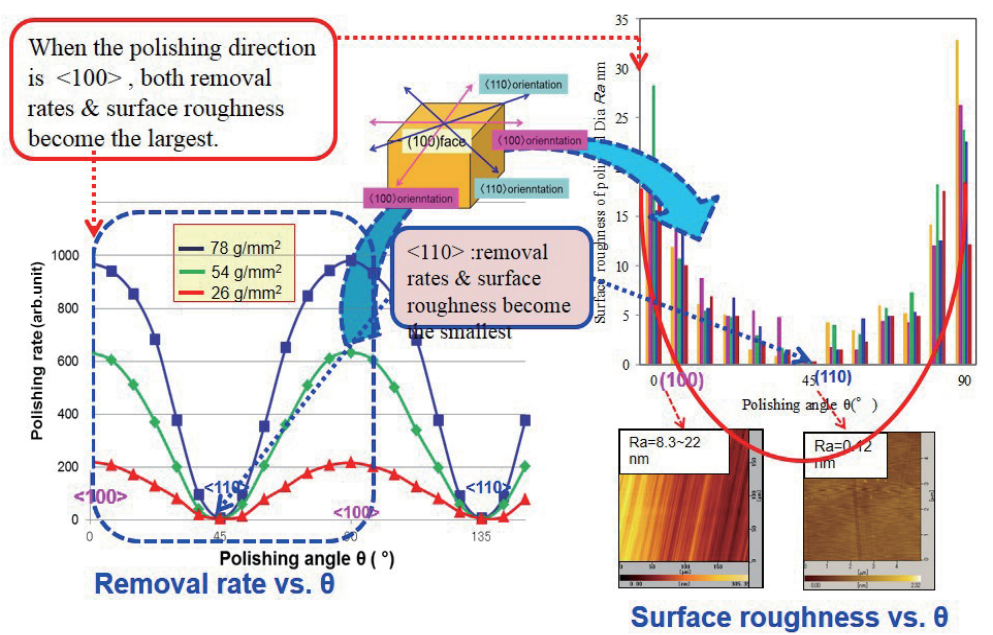

Fig. 5. (Color online) Characteristics of diamond substrate processed by conventional polishing method using diamond abrasives. 


\subsection{Experiment for finishing step}

Sano et al. have studied P-CVM for various substrate materials. ${ }^{(4)}$ Table 1 provides a list of examples, from which we confirmed that P-CVM can process diamond. First, it is necessary to investigate the depth dependence of the processing speed. To conduct a basic processing experiment, a simple atmospheric plasma etching machine was constructed and is shown in Fig. 6. This machine uses a quartz spacer between an electrode for high-frequency bias and a ground electrode. The quartz spacer, which also acts as a plasma observation window, confines the reactive gas in a space $(\sim 2 \mathrm{cc})$ between the electrode and the workpiece. Although P-CVM has been applied to SiC, $\mathrm{GaN}$ and diamond substrates, we will present here the processing characteristics of $\mathrm{SiC}$ substrates.

After mounting a sample in the machine, air in the space between the electrode and the workpiece was purged out and substituted with reactive gas, followed by the immediate start of plasma processing. A rod-shaped electrode was used to produce lineshaped plasma. $5 \mathrm{slm}$ of $0.4 \%$ sulfur fluoride $\left(\mathrm{SF}_{6}\right)$ gas diluted with helium $(\mathrm{He})$ was

Table 1

Examples of removal rates of various materials to be achieved by P-CVM.

\begin{tabular}{lc}
\hline Material & Removal rate $(\mu \mathrm{m} / \mathrm{min})$ \\
\hline Quartz & 170 \\
Solicon & 94 \\
Molybdenum & 36 \\
Tungsten & 32 \\
Silicon carbide $(\mathrm{SiC})$ & 6.4 \\
Diamond & 2.1 \\
\hline
\end{tabular}

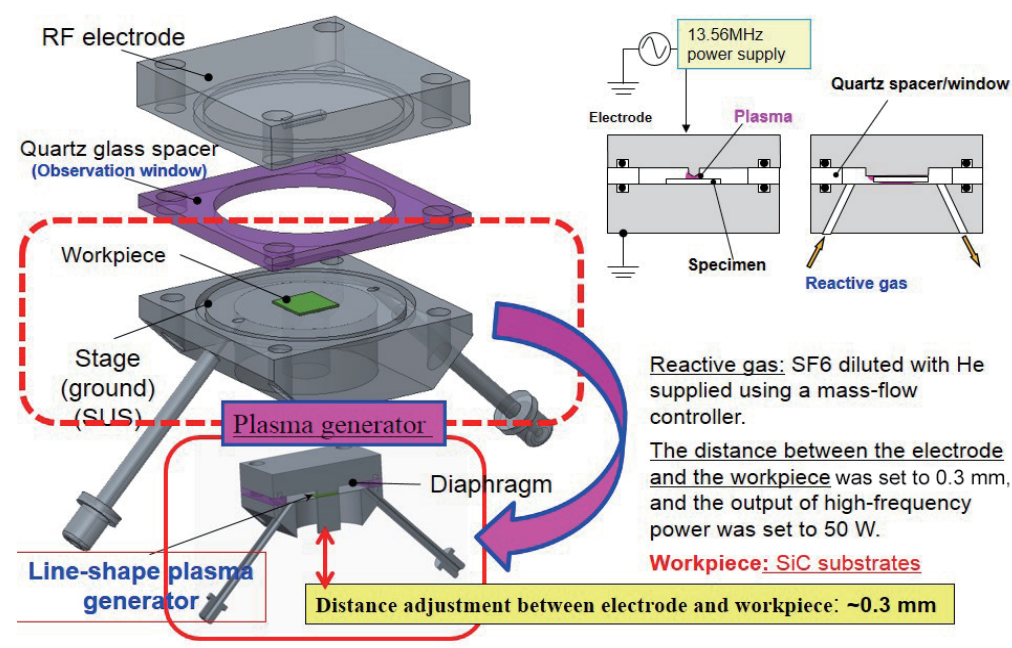

Fig. 6. (Color online) Basic P-CVM apparatus. 
introduced using a mass-flow controller. The gap between the electrode and the sample was approximately $0.3 \mathrm{~mm}$, and the r.f. power was $50 \mathrm{~W}$ at $13.56 \mathrm{MHz}$. One cycle of processing time was $1 \mathrm{~min}$, and after every processing cycle, the surface shape was measured with an interference microscope (Zygo, NewView 3000). An example of the measurement is presented in Fig. 7, in which an example of a cross-sectional view of the processed groove is also shown.

\section{Results of Fundamental Study and Discussion}

\subsection{Creation of pseudoradical area by $f_{s}$ laser irradiation and surface characterization}

A microindentation test was carried out on the samples using a spherical diamond tip $(R=0.5 \mathrm{~mm})$ with a maximum load of $1961 \mathrm{mN}$, a load speed of $35 \mathrm{mN} / \mathrm{s}$, and a load duration of $15 \mathrm{~s}$. Figure 8 shows the relationship between displacement and load for single-crystalline diamond substrates with and without fs laser irradiation. The diamond with laser irradiation showed a 1-2\% larger indentation depth than that without laser irradiation. As expected, the result implies weakened $\mathrm{C}-\mathrm{C}$ bonds in the diamond due to the formation of the pseudoradical area, although some portions showed a displacement increase of less than $1 \%$. Depending on the measured point, the measured data are scattered by $\sim 10 \%$; however, we expect that the surface will become suitable for the next finishing step.

Figure 9 shows a cross-sectional TEM image of the single-crystalline diamond substrate after fs laser irradiation. The image shows two layers at the surface of the

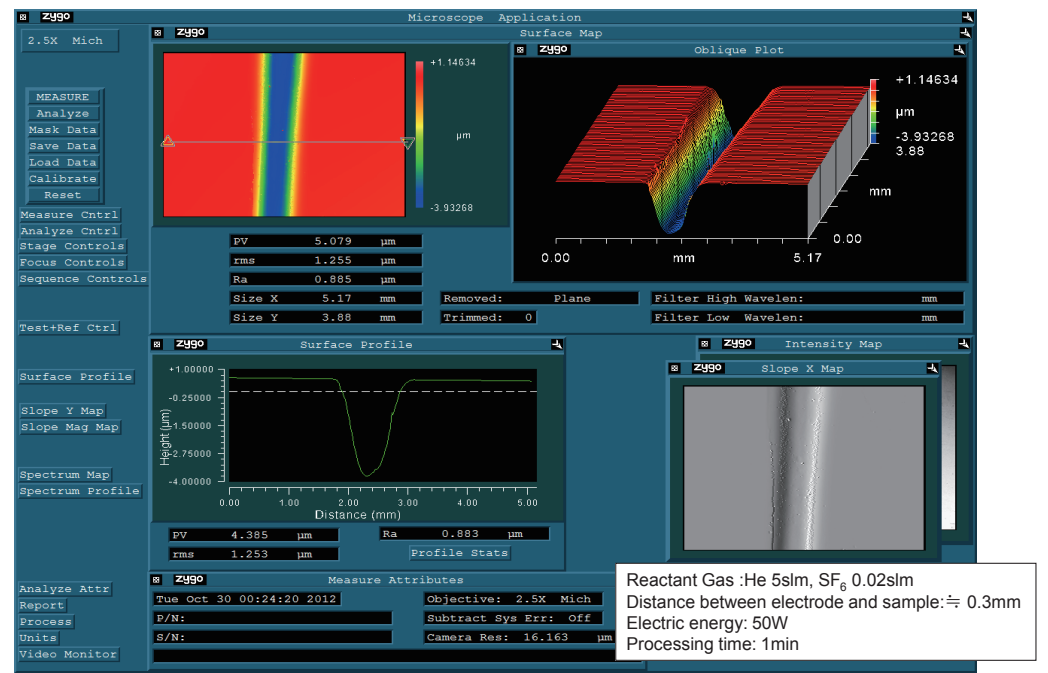

Fig. 7. (Color online) Measurement profile of $\mathrm{SiC}$ substrate, which was linearly processed using a simple P-CVM apparatus. 

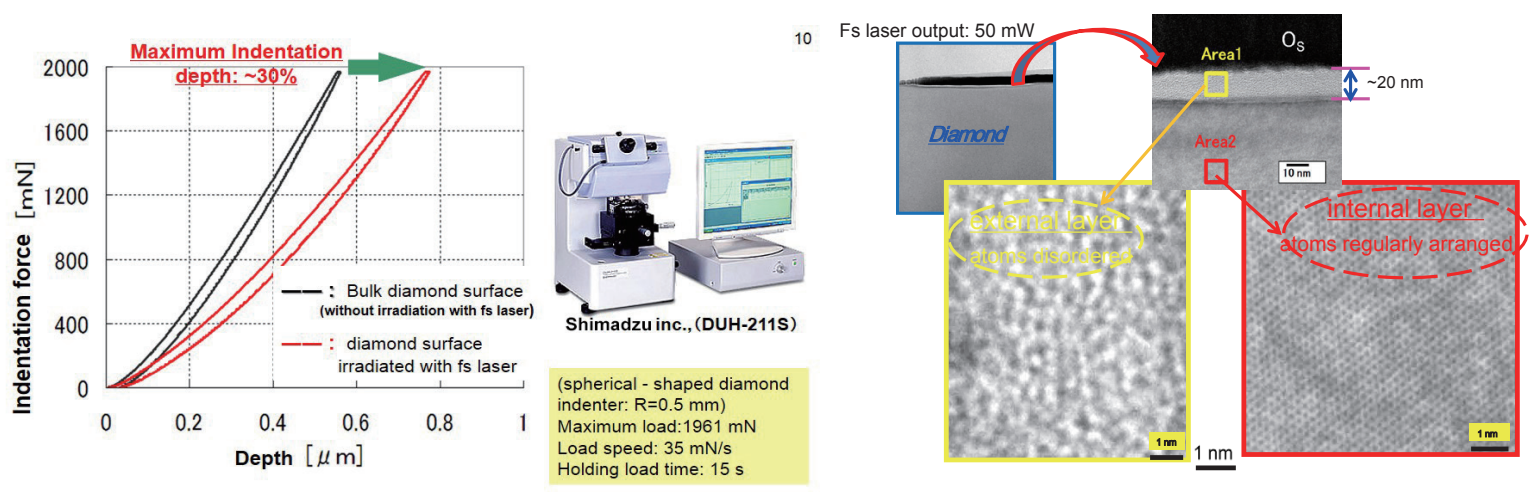

Fig. 8 (left). (Color online) Indentation test on diamond substrates without fs laser irradiation. Fig. 9 (right). (Color online) TEM images (cross sections) of a diamond substrate with fs laser irradiation (pseudoradical sites formed with fs laser).

substrate. The top surface consists of disordered atoms, whereas the inner layer consists of crystallographical ordered atoms. We can conclude that the fs laser irradiation created a pseudoradical area only at the top surface of the substrate. From the cross-sectional TEM image, we estimate the thickness of the modified layer to be approximately $20 \mathrm{~nm}$.

Next, the bonding states of the substrate surface subjected to fs laser irradiation were characterized by Raman spectroscopy. The conditions of laser irradiation are given in Table 2. As shown in Fig. 10, all the substrates subjected to laser irradiation showed a peak originating from the bonding state of graphite $\left(1580 \mathrm{~cm}^{-1}\right)$, whereas the substrate not subjected to laser irradiation did not show the graphite peak. Additionally, when the laser irradiation power decreased, a peak originating from the optical phonon vibration of diamond $\left(1332 \mathrm{~cm}^{-1}\right)$ was observed. Judging from this result, it is concluded that the surface with fs laser irradiation consists of a layer of amorphous carbon on top of the bulk diamond. The cross-sectional TEM and Raman observations confirmed the pseudoradical area created by fs laser irradiation.

\subsection{CMP characteristics of pseudoradical substrates}

CMP using a special oxide slurry was conducted to confirm the effect of the pseudoradical area on process characteristics. Figure 11 shows the variation in surface roughness $(R a)$ as a function of polishing time. As shown in Fig. 10, the roughness of the substrate with fs laser irradiation immediately decreases within 60 min of polishing. The surface roughness of the substrate irradiated with a high-power fs laser, such as Sample 1 , abruptly drops, although it does not change much afterwards. In contrast, the surface roughness of the substrate irradiated with a low-power fs laser, such as Sample 4, reaches the target value $(R a=0.2 \mathrm{~nm})$ earlier. It is necessary to optimize the laser irradiation power considering the entire process since the time needed to reach the roughness of $R a=0.2$ $\mathrm{nm}$ varies depending on the condition of the pseudoradical area. 
Table 2

Conditions of fs laser irradiation.

\begin{tabular}{lccccc}
\hline Sample & 1 & 2 & 3 & 4 & Ref. 1 \\
\hline Output/mW & 747 & 594 & 472 & 375 & Unirradiated \\
\hline
\end{tabular}

Peak due to optical phonon vibration of diamond $\left(1332 \mathrm{~cm}^{-1}\right)$

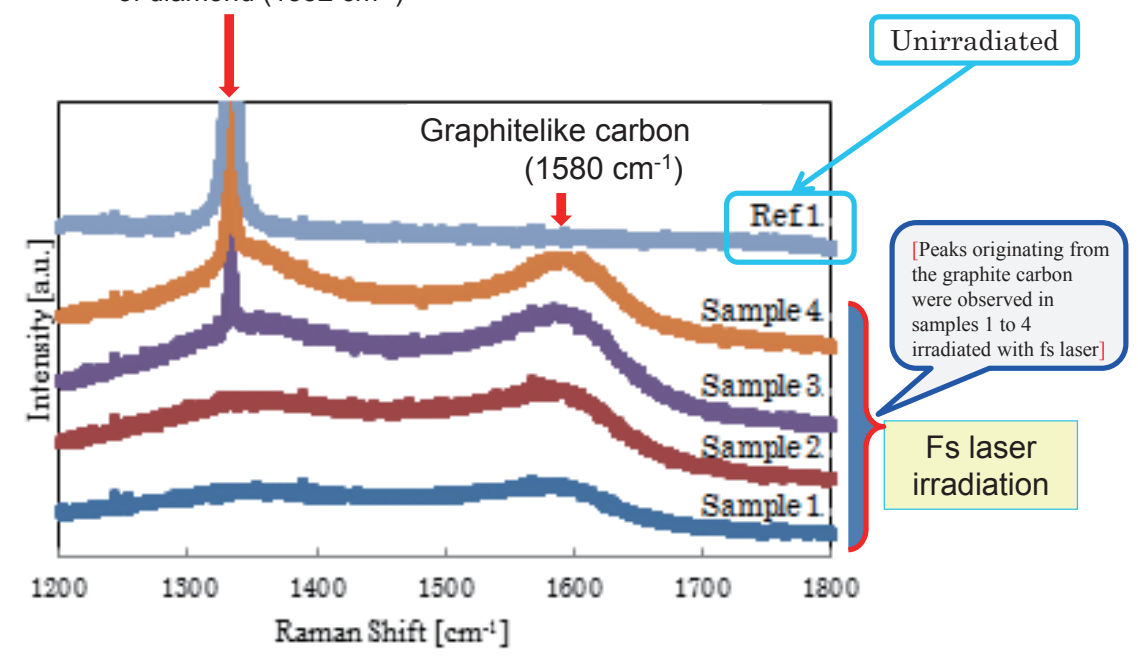

Fig. 10. (Color online) Raman spectra of diamond obtained by fs laser irradiation.

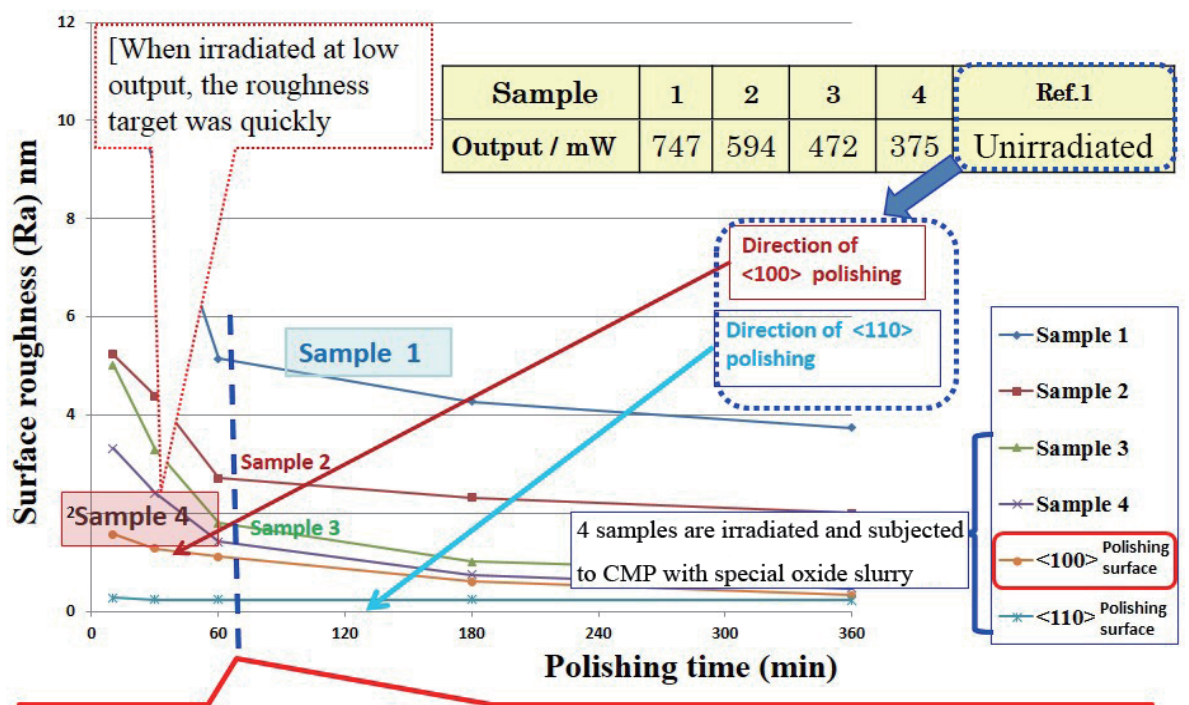

Surface roughness decreased after $60 \mathrm{~min}$ of polishing in the $<100>$ polishing direction in the presence of some damaged layers.

Fig. 11. (Color online) Dependence of surface roughness on polishing time under various conditions of fs laser irradiation. 


\subsection{Results of P-CVM}

The above subsection demonstrated the effect of the pseudoradical area having ultrafine defects created by fs laser irradiation. Other than fs laser irradiation, any method to create ultrafine defects is expected to form a pseudoradical area. Here, we verify the effect of a crystallographically disturbed layer on the top surface (process-induced damaged layer) introduced via coarse processing (grinding). Single-crystalline $\mathrm{SiC}$ and GaN were used as samples.

Figure 12 shows $\mathrm{P}-\mathrm{CVM}$ characteristics of $\mathrm{SiC}$ by comparing a sample having a residual damaged layer from grinding and a stress-free sample. The large slope observed in the early stage of the process for the sample having residual stress indicates a high processing speed. Figure 13 shows the cross-sectional TEM images of the as-ground

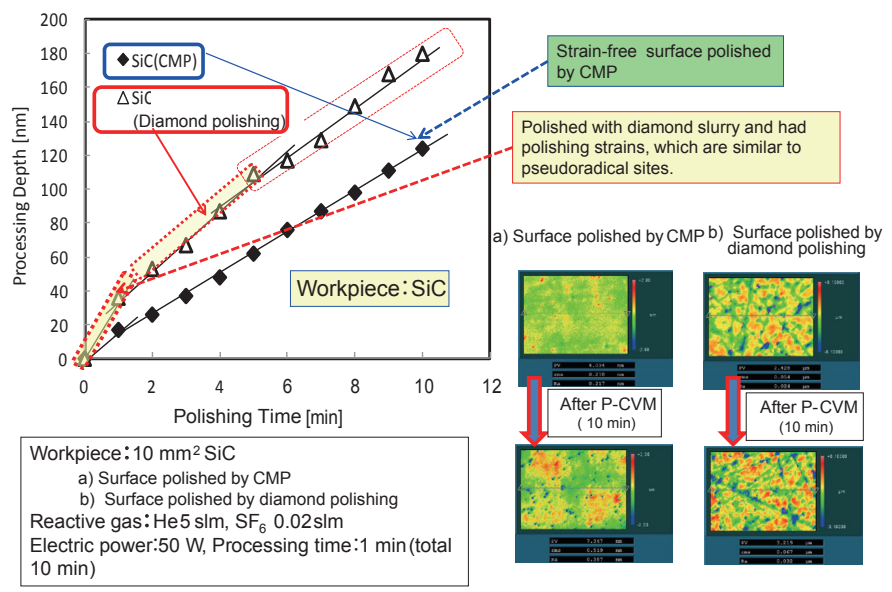

Fig. 12. (Color online) Basic experiment for CMP/P-CVM combined processing machine-P-CVM characteristics of $\mathrm{SiC}$ substrate after two polishing types.

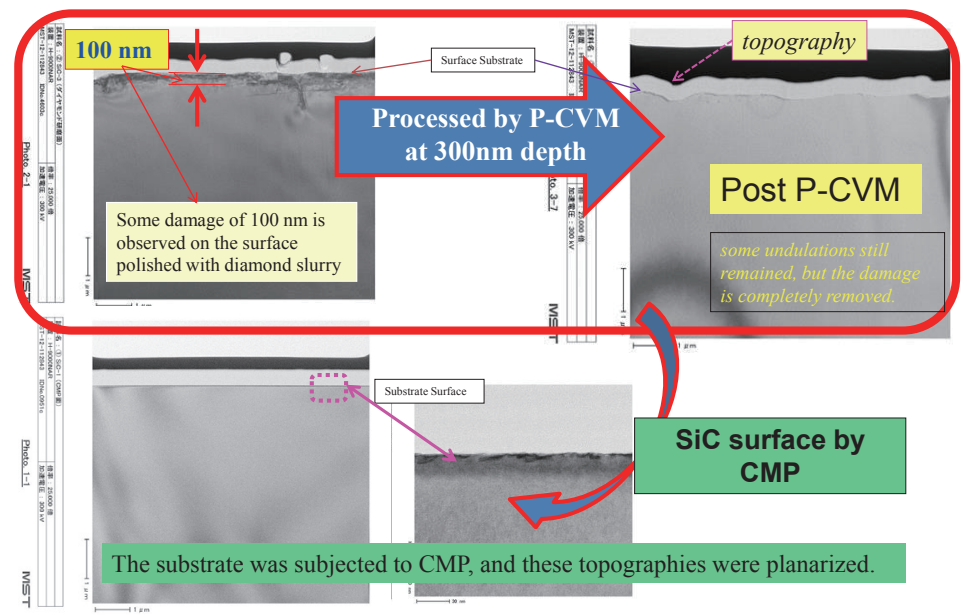

Fig. 13. (Color online) TEM images of SiC surface before and after P-CVM. 
surface before P-CVM and the surface after CMP. The latter sample does not show any crystallographic disorder, whereas the as-ground sample contains a 50-100-nm-thick disordered layer near the surface (the deepest crack extends to $1000 \mathrm{~nm}$ ). When the disordered surface was processed by P-CVM for about $260 \mathrm{~nm}$, the crystallographically disordered portions are all removed, although this process leaves bumps on the process surface. These bumps originate from the difference in local processing speed owing to partially disordered regions.

Similar to the result from fs laser irradiation, we confirmed the increase in processing speed in P-CVM from the effect of the pseudoradical area in which a process-induced damaged layer containing ultrafine defects was created by grinding. Accordingly, we showed the possibility of creating the pseudoradical area followed by P-CVM.

As explained above, we demonstrated the potential of high-efficiency flattening by combining P-CVM and the in situ physical effect. This key concept will lead to a novel fusion processing machine. Figure 14 shows the outline of the machine under construction.

\section{Conclusions}

In this research, we aim to establish systematic knowledge of ultraprecision processing of hard-to-process crystal wafers for next-generation green devices, and design and develop a high-efficiency, high-quality process to contribute to an early commercialization of $\mathrm{SiC}, \mathrm{GaN}$ and diamond-based devices demanded for a low-carbon society.

Upon designing an ultrahigh-precision process for hard-to-process materials, we divided the process into two steps: a pretreatment step and a finishing step. In the pretreatment step, a pseudoradical area was formed by introducing ultrafine defects to control the surface condition suitable for finishing. In the finishing step, we attempted

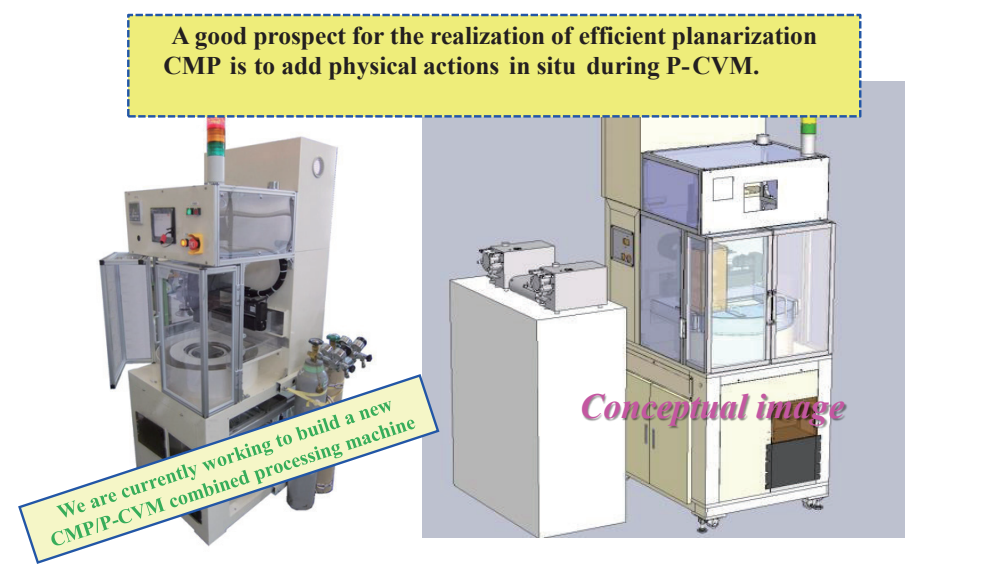

Fig. 14. (Color online) Conceptual image of a new CMP/P-CVM combined processing machine. 
to combine the closed-chamber CMP and P-CVM methods. To evaluate the concept of the second step, a fundamental study needed to design and prototype the machine was conducted.

As a method of creating a pseudoradical area, fs laser irradiation and coarse processing (grinding), which leaves a crystallographically disordered layer (processinduced damaged layer), were considered. Microindentation test, cross-sectional TEM observation, Raman spectroscopy, and X-ray photoelectron spectroscopy (XPS) were used to confirm the pseudoradical area at the uppermost surface. Also, we studied the characteristics of the pseudoradical substrates subjected to CMP and P-CVM. $\mathrm{P}-\mathrm{CVM}$ showed an increased processing rate for the pseudoradical substrates having microdefects. In addition, it was demonstrated that CMP could efficiently flatten the surface having a moderate level of crystallographic disorder. It will be necessary to optimize the degree and depth of crystallographic disorder in the pseudoradical area in the future.

On the basis of the results presented here, we are starting to prototype the innovative $\mathrm{CMP} / \mathrm{P}-\mathrm{CVM}$ fusion processing machine that can selectively flatten protrusions of hard-to-process materials by conducting P-CVM while continuously creating a pseudoradical area through an in situ physical effect such as CMP. We aim to establish the ultraprecision process by understanding the process mechanism and optimizing the process using the innovative CMP/P-CVM fusion processing machine on hard-to-process materials.

\section{Acknowledgements}

This research was supported by a Grant-in Aid for Scientific Research (S) from the Japan Society for the Promotion of Science (No. 24226005). The authors thank Dr. Tsukamoto, Mr. Ji, Mr. Wang and Mr. Komai of Kyusyu University, Mr. Shiozawa and Mr. Okada of Osaka University, Mr. Takeda of Namiki Precision Jewel Co., Ltd., and Dr. Takahashi of Disco Co., Ltd. for the support of the experiments.

\section{References}

1 Nikkei Electronics (2011.12.21) p. 67.

2 W. Saito, I. Omura, T. Ogura and H. Ohashi: Solid State Electron. 48 (2004) 1555.

3 T. Doi, I. D. Marinescu and S. Kurokawa: Advances in CMP Polishing Technologies (Elsevier, New York, 2012) Chap. 5, p. 40.

4 Y. Sano, M. Watanabe, T. Kato, K. Yamamura, H. Miura and K. Yamauchi: Mater. Sci. Forum 600-603 (2009) 847.

5 T. Doi: Details of Semiconductor CMP Technology (Kogyo-chosakai Publishing Co., Tokyo, 2001) (in Japanese).

6 T. Doi, A. Philipossian and K. Ichikawa: Electrochem. Solid State Lett. 7 (2004) G160.

7 Y. Nakahama, N. Kanetsuki, T. Funaki, M. Kadono, Y. Sano, K. Yamanura, K. Endo and Y. Mori: Surf. Interface Anal. 40 (2008) 1566. 